\title{
DRIVER BEHAVIOUR STUDIES IN THE MOTORWAY OPERATIONS PLATFORM GRANT
}

\author{
Mark Brackstone and Mike McDonald \\ Transportation Research Group \\ Dept. of Civil and Environmental Engineering \\ University of Southampton \\ Southampton, Hants, U.K. \\ Email: mab6@soton.ac.uk,mm7@soton.ac.uk
}

Summary: This paper will report on a four-year project being undertaken in the U.K., which intends to address the causative mechanisms of motorway congestion, and how these may be overcome by the use of in-vehicle Intelligent Transport Systems (ITS). The project comprises five studies, two focussing on driver behaviour and performance, and three on microscopic simulation and road operations. This paper will provide an overview of progress made and work in progress in the former of these topics, in particular:

i) Phase 1: an instrumented vehicle study collecting microscopic time series on how drivers behave in slow moving dense traffic. An overview of results from this phase will be presented.

ii) Phase 2: to be initiated in late 2001, looks to examine how drivers behave when faced with the requirement for an emergency deceleration. The study will use a combination of a surrogate vehicle/test track approach and a fixed base driving simulator study, in order to examine the advantages of the differing methodologies and (if validity is proven) to increase database size.

A brief review will be given of the intended use of outputs from these studies in subsequent simulation modelling studies to be undertaken in future years.

\section{BACKGROUND}

Driver behaviour has seen a resurgence of interest over the last five years, as it has increasingly become apparent that a detailed understanding of how we drive is essential for both the design and assessment of new driving aids (such as Adaptive Cruise Control, e.g. Marsden et. al., 2001), and for the development of microscopic simulation models that may be used to assess the effect of ITS on traffic systems as a whole Although many studies are now underway (e.g. Saad and Villame, 1996), and indeed large scale normative databases are now within our grasp through the use of instrumented vehicles, interest has focussed on commonly observed features of behaviour, particularly on motorways, where studies of vehicle following for example are the norm (e.g. McDonald et. al., 1999). Two areas however have yet to be examined in detail - the behaviour of a driver when faced with congestion, and the behaviour of a driver in an 'emergency' - which are vital elements in being able to correctly describe the onset and growth of congestion. In order to address these needs, two driver behaviour studies are being undertaken in the U.K. as part of 
the Platform grant on Motorway Operations, in an attempt to at least provide some initial understanding of this behaviour (Brackstone and McDonald, 2001).

\section{PHASE 1: BEHAVIOUR IN CONGESTION}

It has long been held that driver behaviour when faced with dense traffic is somehow different from that which occurs at low levels of traffic flow. For example, it has been suggested that following distances may become shorter and reaction times decrease as drivers 'bunch up' and maybe ready themselves to react to potential shockwaves that may form, necessitating faster decelerations. These assertion however, are on the whole based on conjecture and it has been the intent of this study to quantify these changes, though using an instrumented vehicle to measure driver characteristics.

\section{Data Collection and Analysis}

Initial data collection took place in Oct. 2000 in the evening peak on the A35 dual carriageway, westbound, heading out of Southampton in the U.K.. The vehicle used is equipped with an automotive radar and is able to measure the distance between the equipped vehicle and (in this case) a following vehicle (Brackstone et. al., 1999). All told, 81 time series were collected over seven peak periods with an average length of $127 \mathrm{sec}$. (an example of which is given in Figure 1). Analysis of the data has been performed using three approaches highlighted below.

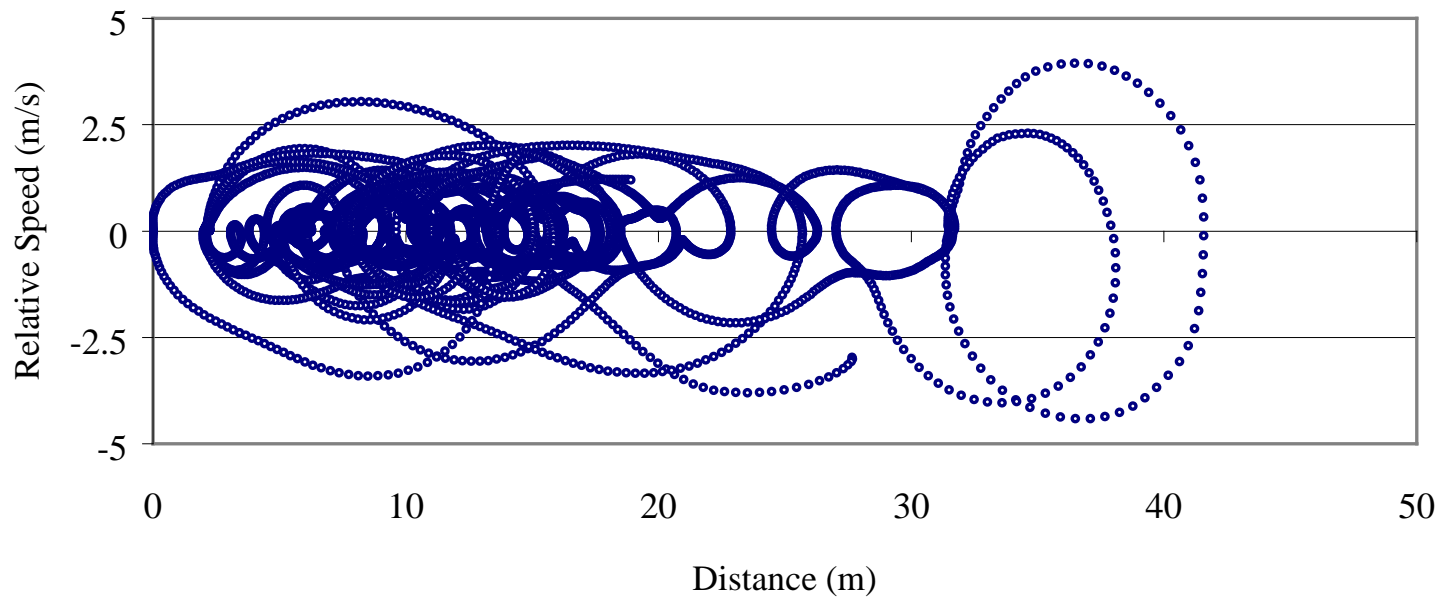

Figure 1: A ‘spiral function’ of following distance vs relative speed.

\section{Findings}

\section{Following Distance}

Deterministic and probabilistic relationships between speed and desired following distance have been established both for individuals and the observed population (Fig. 2). In this case a number of simple distance keeping models have been fitted to the data with the best fit being obtained by a power relationship between the following distance ' $D X$ ' and the speed ' $v$ ' $\left(D X=3.3 v^{0.62}, r^{2} \sim 0.76\right)$. 


\section{Reaction Times}

Through examination of the speed profile of the lead and following vehicle it has been possible to measure four specific reaction points describing the time taken for the following vehicle to react to changes in the lead vehicle speed, these have been classified according to the formalism introduced by Ozaki (1993), and are summarised in Table 1:

Type A; Time from DV (relative speed) $=0$ to the next point at which $\mathrm{a}_{\mathrm{f}}$ (follower acceleration) $<0$

Type B: Time from max. negative DV to max negative $a_{f}$ Type C: Time fromDV $=0$ to the next point at which $\mathrm{a}_{\mathrm{f}}>0$ Type D: Time from max. positive DV to max. positive $\mathrm{a}_{\mathrm{f}}$.

The reaction times can clearly be split into two groups, A and C, both characterised by a higher average and standard deviation (not significantly different from each other, $\mathrm{p}=0.61$ ), and $\mathrm{B}$ and $\mathrm{D}$ both with a lower reaction time (again not statistically different from each other, $\mathrm{p}=0.12$ ). The two groups are however significant from each other with a grouped ANOVA analysis revealing a significant difference at the $\mathrm{p}<0.01$ level $(\mathrm{F}=42.8)$.

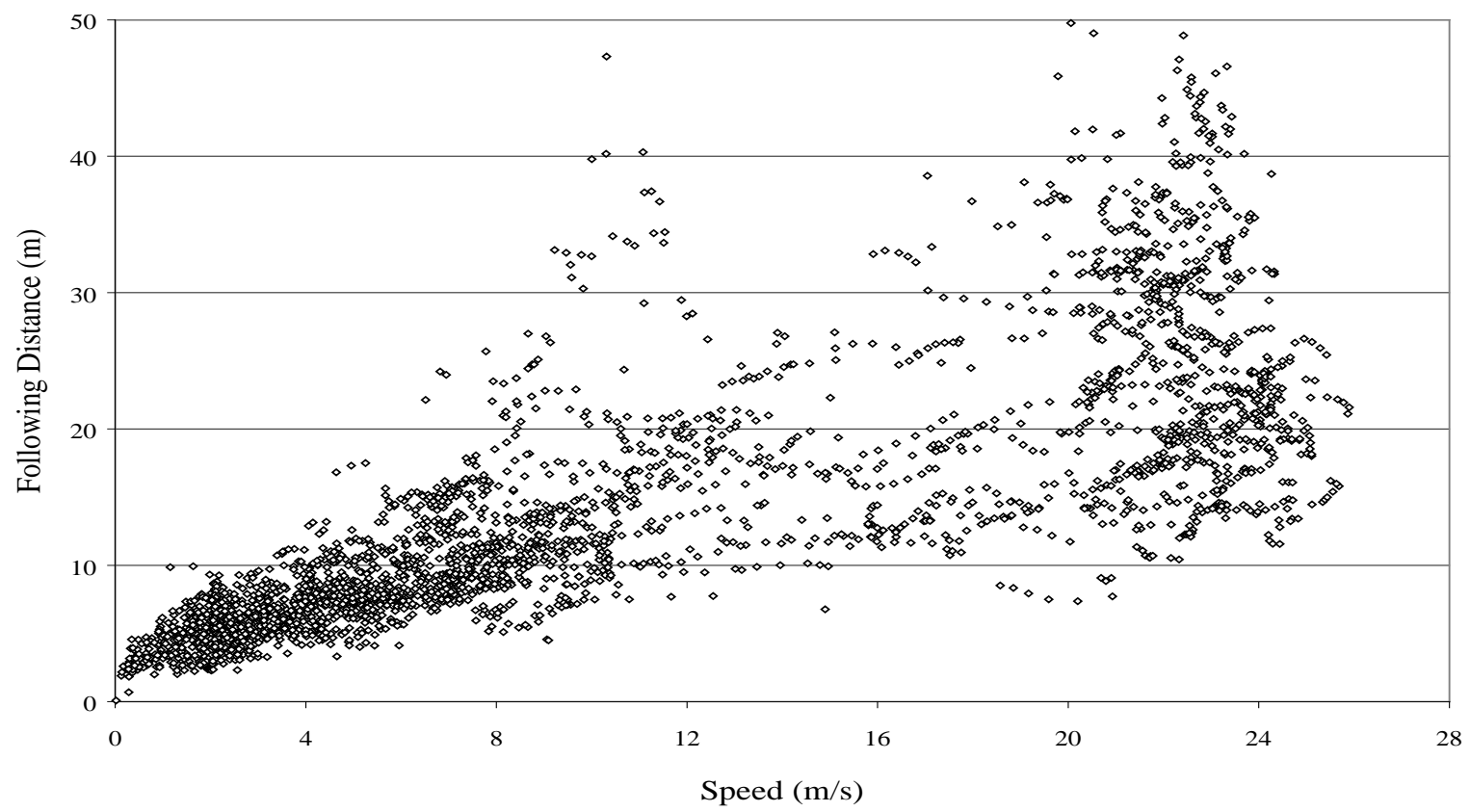

Figure 2: Following Distance vs Speed for all observed vehicles in stable following (magnitude of relative speed less than $0.5 \mathrm{~m} / \mathrm{s}$ ).

\begin{tabular}{|l|l|l|l|l|}
\hline & \multicolumn{4}{|l|}{ Type } \\
\hline & $\mathrm{A}(\mathrm{N}=268)$ & $\mathrm{B}(\mathrm{N}=300)$ & $\mathrm{C}(\mathrm{N}=271)$ & $\mathrm{D}(\mathrm{N}=290)$ \\
\hline Average (sec.) & 2.03 & 1.44 & 2.14 & 1.34 \\
\hline St. Dev.(sec.) & 2.38 & 0.78 & 2.50 & 0.77 \\
\hline
\end{tabular}

Table 1: Average and standard deviations of reaction times. 


\section{Error Margins}

We may define error margin to be i) the error present in maintaining a desired distance, and ii) the mismatch between average following headway and actual reaction time. In the first of these cases we have compared the standard deviation of the observed headways at a number of discrete levels with the average headway at that level. In the second case we have quantified the difference between average observed headway and an average reaction time (averaging across speed and all the types detailed earlier). Results are shown in table 2.

\begin{tabular}{|l|l|l|l|l|l|l|l|}
\hline & \multicolumn{2}{|l|}{ Speed range (m/s) } \\
\hline & $<4$ & $4-8$ & $8-12$ & $12-16$ & $16-20$ & $20-24$ & $>24$ \\
\hline $\begin{array}{l}\text { Ave. \% error in distance } \\
\text { keeping }\end{array}$ & 59 & 29 & 43 & 36 & 39 & 35 & 23 \\
\hline $\begin{array}{l}\text { Error between ave. } \\
\text { headway and reaction time } \\
\text { a)(sec.) }\end{array}$ & +1.09 & -0.11 & -0.24 & -0.45 & -0.60 & -0.55 & -0.88 \\
\hline b) \% & 61.2 & 106.8 & 116.2 & 135.4 & 153.6 & 147.0 & 204.8 \\
\hline
\end{tabular}

Table 2: Error margin summary results.

\section{Discussion and Ongoing Work}

The analysis in the above sections has shown that the gaps kept by drivers at low speeds are just as close (proportionally) as those that would be maintained at higher speeds, and that if anything drivers would not seem to drive at a constant time headway (a result that has clear implications for automated low speed driving which relies on such a constant relationship). There is a large degree of variation in these following distances too, with cycles of approach and backing away seemingly characterised by two distinct reaction times, giving a following process where the driver operates consistently outside of his time headway safety margin (above $8 \mathrm{~m} / \mathrm{s}$ drivers are operating at at least a quarter of a second below their reaction times). One key question remaining is that of the relationship between the reaction time and the criticality of the action required (characterised by TTC - time to collision, or optic flow) and the next stage of analysis will focus on this question.

Additional work is also to be performed in examining whether the general findings above are transferable to individuals, with six test subjects being asked to drive the vehicle and observations made of their behaviour in a 2x2 condition study. Condition A - type of road, Motorway vs A road (similar traffic conditions but differing geometry and speed limits), Condition B - type of vehicle being followed (car vs high sided van, restricting forward visibility). Additionally an attempt will be made to see if any of the drivers characteristics correlate with a number of simple personality measures assessed through the use of a Sensation Seeking Scale (SSSV).

\section{PHASE 2: DRIVER BEHAVIOUR IN AN EMERGENCY}

The second phase is attempting to formulate a model of how drivers react when faced with a harshly decelerating vehicle, as, in such cases a driver may not decelerate in accordance with a set following 
relationship but may instead react instinctively, producing a rapid deceleration followed by a more measured response once a potential collision situation is averted. Although studies exist which have quantified differing types of reaction time according to the urgency of the situation (measured for example by TTC - Time To Collision), no data is available on the braking profiles that drivers use. (For a review see Fambro et. al., 1999). This has particularly severe consequences on our ability to conduct platoon collision studies, where risk exposure to a number of following drivers is examined as a function of the deceleration of a platoon leader (e.g. Touran et. al. 1999), where it has been found that results are extremely sensitive to the form of the brake reaction model used.

The study, which is to be performed toward the end of 2001 will use a combination of two methods. Firstly, a surrogate vehicle/test track approach (e.g. Fig. 3), where subjects will be asked to follow a towed impact resistant target in an instrumented vehicle, with the driver of the vehicle towing the surrogate target performing several braking manoeuvres (larger than $-4 \mathrm{~m} / \mathrm{s}^{2}$ ). In support of this method these experiments are to be replicated on a fixed base driving simulator in order to both examine the advantages of the differing methodologies and (if validity is proven) to increase the size of the database.

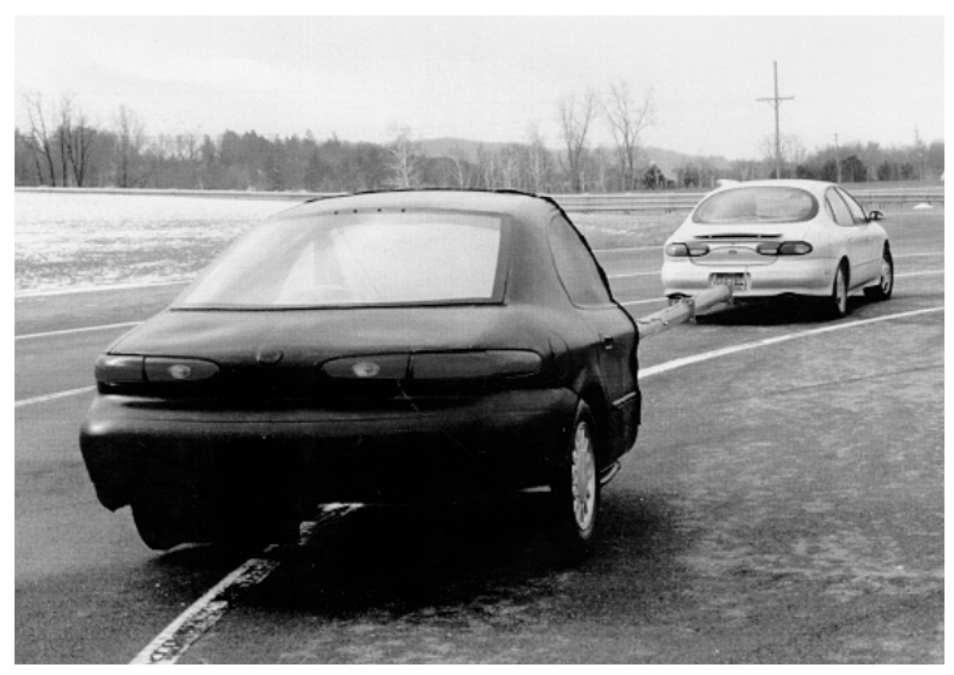

Figure 3: An example of a Surrogate vehicle test (Photo. appears courtesy of Ford R\&D)

\section{ACKNOWLEDGEMENTS}

Work reported in this paper has been funded by the EPSRC in the U.K. under contract number GR/M94410. Support for the instrumented vehicle continues to be provided by TRW-Lucas and the University of Southampton. Further details on this project may be obtained at:

http://www.soton.ac.uk/ trgwww/research/platform/platform.htm

\section{References}

Brackstone, M. and McDonald, M. (2001). Barriers to Motorway Traffic Operations, and their Potential Solution. Proc. of the IEEE Intelligent Vehicles Symposium 2001, Tokyo.

Brackstone, M. McDonald, M. and Sultan, B. (1999). Dynamic Behavioural Data Collection Using an Instrumented vehicle. Transpn. Res. Rec., 1689, pp 9-17. 
Fambro, D., Koppa, R, Picha, D. and Fitzpatrick, K. (1999). Driver Perception - Brake Response in Stopping Sight Distance Situations. Transpn. Res. Rec., 1628. TRB, Washington D.C., U.S.A.

McDonald, M., Brackstone, M., Sultan, B. and Roach, C. (1999). Close Following on the Motorway: Initial Findings of an Instrumented Vehicle Study. Vision in Vehicles VII. pp 381-9. Elsevier, Netherlands.

Marsden, G., McDonald, M. and Brackstone, M. (2001). Towards and Understanding of Adaptive Cruise Control. T.Res. C, 9(1), pp 33-51.

Ozaki, H. (1993). Reaction and Anticipation in the Car Following Behaviour. Proc. of the Thirteenth International Symp. on Traffic and Transportation Theory 2 pp 349-366.

Saad, F. and Villame, T. (1996). Proc. of the 3rd Ann. World Cong. on ITS. Orlando, FL, USA.

Touran, A., Brackstone, M., and M. McDonald. (1999). A Collision Model for Safety Evaluation of AICC. Acc. Analy. and Prev., 31(5), pp 567-78. 